\section{Questões metodológicas para a investigação dos efeitos da poluição do ar na saúde}

\author{
Methodological issues of the \\ research on the health effects \\ of air pollution
}

\author{
Hermano Albuquerque de Castro \\ Pneumologista \\ Pesquisador do CESTEH/ENSP/FIOCRUZ \\ Rua Leopoldo Bulhões, 1480 \\ Manguinhos - Rio de Janeiro, CEP: 21041-210 \\ Tel.: 55-021-25982682 \\ castro@ensp.fiocruz.br
}

\section{Nelson Gouveia}

Epidemiologista

Professor do Departamento de Medicina Preventiva Faculdade de Medicina

Universidade de São Paulo

José A. Escamilla-Cejudo

Epidemiologista

Informações para Política de Saúde

OPAS - Organização Panamericana da Saúde, Brasil

\section{Resumo}

Junto com o processo de urbanização e o crescimento das cidades tem havido também um aumento da contaminação do meio ambiente e, entre outros agravos, a poluição do ar tem tido posição de destaque, visto atingir grandes parcelas da população e possuir diversos efeitos na saúde que vêm sendo estudados em maior profundidade nos últimos anos. Este artigo discute algumas questões metodológicas envolvidas na avaliação dos efeitos na saúde associados à poluição do ar.

A primeira questão que se coloca é a dos indicadores epidemiológicos em saúde ambiental. Esses indicadores podem ser vistos como medidas que sumarizam alguns aspectos da relação entre saúde e meio ambiente. A metodologia de indicadores proposta pela Organização Mundial de Saúde para o desenvolvimento de ações de saúde ambiental vem sendo aplicada em nosso meio no que diz respeito à qualidade do ar. Um segundo aspecto refere-se à avaliação da exposição à poluição do ar, talvez o aspecto mais difícil nos estudos nesta área. Questões acerca dos diferentes microambientes (externos e internos) são relevantes e devem, sempre que possível, ser levados em conta de maneira a minimizar as fontes de erro em relação à correta exposição à qual os indivíduos estão expostos. Por último, é apresentada uma breve discussão dos principais tipos de estudos epidemiológicos utilizados para examinar a associação entre a exposição à poluição do ar e os efeitos na saúde. As vantagens e desvantagens dos diversos tipos de estudos e suas principais aplicações são discutidas à luz da metodologia epidemiológica.

Enfatizamos que estas não são as únicas questões relevantes quando se procura avaliar os efeitos deletérios da poluição do ar, mas representam algumas das mais importantes. Todavia, para que sejam construídas políticas de saúde ambiental coerentes e eficazes, é preciso realizar estudos bem delineados sobre a relação entre poluição do ar e saúde, levando-se em conta essas questões. Desse modo, pode-se garantir uma melhor qualidade na informação que será utilizada pelos gestores que implementarão essas políticas.

Palavras-chave: Poluição do ar. Efeitos na saúde. Indicadores em saúde ambiental. Avaliação da exposição. Estudos epidemiológicos. 


\section{Abstract}

Urbanization and the growth of cities have contributed to increase the load of contaminants in the environment. Among contaminants, air pollutants remain a major cause of concern due to their capacity to affect large populations and the fact that several adverse health effects associated with exposure to air pollution have been described in the past few years. This article reviews and discusses some methodological issues involved in the assessment of these health effects.

The first issue relates to environmental health indicators (EHI). These indicators can be seen as measures that summarize aspects of the relationship between health and the environment. The EHI methodology proposed by the World Health Organisation for the development of environmental health actions has been applied in the area of air quality. A second aspect relates to the assessment of exposure to air pollution. This is perhaps the most difficult task for studies in this area. Issues concerning different microenvironments (indoor and outdoor) are relevant and should be taken in to account in order to minimize the errors associated with the correct assessment of exposure to air pollutants. Lastly, a brief discussion of the main epidemiological study designs used to examine the association between air pollution and health effects is presented. The advantages and disadvantages of the main study types and their main applications are discussed.

These are not the only essential issues when studying the deleterious health effects of air pollution on health. However, in order to have sound environmental health policies one needs well designed and conducted epidemiological studies taking in to account these main issues. In this manner, better information will be available to those involved in the decision-making process regarding air quality in our cities.

Key words: Air pollution. Health effects. Environmental health indicators. Exposure assessment. Epidemiological studies.

\section{Introdução}

A poluição do ar representa hoje um dos maiores problemas de Saúde Pública, afetando a saúde dos seres humanos, de outros animais e das plantas. O rápido avanço tecnológico do mundo moderno trouxe consigo um aumento na quantidade e na variedade de poluentes eliminados na atmosfera, prejudicando de maneira muito séria a qualidade de vida em nosso planeta.

No ano de 1952 assistiu-se, em Londres, um dos primeiros grandes episódios de poluição do ar do mundo moderno, com conseqüências dramáticas para a saúde da população, entre elas um aumento enorme na mortalidade. Desde então, a sociedade tem discutido mecanismos para a redução de poluentes e vem buscando conhecimentos, em todos os campos, para enfrentar a poluição do ar. Mesmo assim, em 1990, foram lançados na atmosfera terrestre, cerca de 100 milhões de toneladas de óxidos de enxofre (SOx), 68 milhões de toneladas de óxidos de nitrogênio (NOx), 57 milhões de toneladas de Material Particulado (MP) e 177 milhões de monóxido de carbono (CO) ${ }^{1}$.

Posterior à constatação do aumento da mortalidade em Londres, após o impacto da poluição, diversos e diferentes estudos foram desenvolvidos demonstrando a relação entre morbi-mortalidade cardio-respiratória e os poluentes do ar em diferentes concentrações. Tais estudos têm contribuído para definir padrões de aceitabilidade nos níveis de concentração dos contaminantes mais comuns.

Ainda assim, estudos epidemiológicos mais abrangentes vão se desenvolvendo em diferentes capitais e cidades do mundo, no sentido de se estabelecer padrões de qualidade do ar. Alguns destes estudos mostram aumento de morbidade em ambientes com concentrações mínimas de poluentes, causando principalmente agravos em idosos e crianças.

Entre os objetivos deste artigo estão a discussão e a apresentação dos principais indicadores de poluição do ar, tendo como matriz o modelo de indicadores da Organi- 
zação Mundial de Saúde. Serão abordadas questões vinculadas à avaliação da exposição dos poluentes e serão mostrados ainda os principais desenhos de estudos epidemiológicos para a compreensão da relação entre a qualidade do ar e a saúde.

\section{Indicadores Epidemiológicos em Saúde Ambiental}

De acordo com a Organização Panamericana de Saúde (OPAS), indicador ambiental pode ser entendido como uma expressão da relação entre a saúde e o ambiente e pode ser definido como "um valor agregado a partir de dados e estatísticas, transformado em informação para uso direto dos gestores. $e$ deste modo contribuindo para aprimoraro gerenciamento e a implementação de políticas" $^{2,3}$.

Os indicadores são úteis para a vigilância ambiental em saúde, e sua escolha contribui para uma ação eficaz no controle dos poluentes, orienta a prática e fornece elementos para a tomada de decisões.

De um modo geral, os indicadores utilizados para a decisão no controle da qualidade do ar devem apresentar algumas características que facilitem seu uso e interpretação:

- Primeiro, devem corresponder à realidade local e ou regional, como por exemplo, medir poluentes que façam parte da matriz dos poluentes locais. Esta informação pode ser conseguida a partir do conhecimento do modelo socioeconômico local e/ou regional;

- Segundo, os indicadores devem ter sustentabilidade científica, ou seja, a informação e a interpretação do indicador devem ter comprovada validade científica;

- Terceiro, o indicador deve ser de fácil manuseio pelo usuário local e mostrar uma fácil compreensão do seu papel na relação entre saúde e ambiente;

- Quarto, o indicador deve, sempre que possível, compor uma matriz global no sistema de informação, em todos os seus níveis.

Atualmente, para o controle da qualidade do ar, tem-se preferido a utilização de poucos indicadores que permitam uma informação completa do quadro local, regional e nacional.

O indicador de saúde ambiental corresponde à intercessão entre a relação de danos na saúde e o ambiente. Encontram-se ainda indicadores ambientais com possíveis impactos na saúde e que precisam ser mais bem estudados e esclarecidos. E, por fim, existem indicadores de saúde com possíveis causas ambientais e que também necessitam de estudos para uma melhor compreensão do nexo de causalidade entre o ambiente e a saúde.

Neste artigo optou-se por apresentar o modelo proposto pela Organização Mundial de Saúde (OMS) e que atualmente vem sendo utilizado pela Fundação Nacional de Saúde (FUNASA), como proposta para o desenvolvimento da vigilância ambiental em saúde, no que diz respeito ao controle da qualidade do ar.

De acordo com a OMS, a proposta da construção dos indicadores ambientais em saúde é uma adaptação da estrutura de Pressão-Situação-Resposta do modelo desenvolvido pela Organização para a Cooperação Econômica e o Desenvolvimento (OECD), tendo por base o modelo encomendado pelo Governo Canadense ${ }^{2}$.

Esta estrutura estabelece uma relação de causa e efeito no desenvolvimento da matriz de indicadores. A estrutura é composta por Força Motriz, Pressão, Situação, Exposição, Efeito e Ações. O modelo permite ao gestor ter uma boa visão das causas macro, onde se inicia o processo poluidor até os efeitos no indivíduo. Para cada um dos itens do modelo serão identificadas as diversas matizes que compõem o padrão de poluentes do ar e seus efeitos. Segundo a OMS, "este é o modelo através do qual as forças motrizes geram pressões que modificam a situação do ambiente e, em última análise, a saúde humana, por meio das diversas formas de exposição, onde as pessoas entram em contato com o meio ambiente, causando os efeitos na saúde. Várias ações podem ser desenvolvidas em diferentes pontos da cadeia"'.

$\mathrm{O}$ modelo precisa ser compreendido 
como uma possibilidade para o seu uso na gestão de riscos e agravos. Não se limita apenas à causa direta e aos efeitos na saúde, mas proporciona uma visão macro, possibilitando ações de vigilância exeqüíveis em saúde ambiental. Neste sentido, as ações no âmbito da força motriz são mais eficazes do que as ações sobre os efeitos gerados pelos poluentes.

O resultado deste modelo construído no segundo curso de Vigilância Ambiental em Saúde, realizado no Estado de São Paulo, com o patrocínio da Fundação Nacional de Saúde (FUNASA) e o Instituto Brasileiro de Geografia e Estatística (IBGE) foi apresentado por Eloísa Domingues no II Seminário Nacional de Saúde e Ambiente. (Quadro 1)

\section{Indicadores de força motriz}

Os indicadores neste âmbito estão estreitamente relacionados ao modelo econômico da região. Ele propicia uma visão macro sobre os problemas que possam gerar danos ao ambiente e a saúde. O desenvolvimento econômico funciona como a mola propulsora dos elementos que compõem a força motriz da região. O crescimento populacional, o modelo habitacional e o uso de tecnologias na região definem os padrões de poluição. Cidades metrópoles tendem a ter sua sustentação baseada em serviços e indústrias, conferindo um padrão de poluição viária e industrial. Cidades que possuem modelos agrícolas baseados em queimadas

Quadro 1 - Lista mínima de indicadores para o monitoramento da qualidade do ar na Bacia do Alto Rio Tietê - SP Chart 1 - Minimun list of indicators for monitoring air quality in the Alto Tietê River Basin - SP

\begin{tabular}{|c|c|c|}
\hline & INDICADORES & AÇÕES \\
\hline FORÇA MOTRIZ & $\begin{array}{l}\text { - Política de incentivo à construção } \\
\text { de veículos automotores } \\
\text { - Política de transporte à base de } \\
\text { combustível derivado de petróleo } \\
\text { - Ausência de planejamento viário } \\
\text { urbano } \\
\text { - Densidade populacional/ano }\end{array}$ & $\begin{array}{l}\text { - Intervenção nas políticas públicas } \\
\text { - Planejamento e controle da } \\
\text { distribuição espacial da população }\end{array}$ \\
\hline PRESSÃO & $\begin{array}{l}\text { - Quantidade de veículos/tipo de } \\
\text { combustível usado } \\
\text { - Tecnologia do veículo/tamanho } \\
\text { da frota } \\
\text { - Qualidade da rede viária } \\
\text { - Quantidade de veículos/dia } \\
\text { - Volume (t) das substâncias lançadas } \\
\text { por plantas industriais }\end{array}$ & $\begin{array}{l}\text { - Redução dos valores de emissão } \\
\text { - Determinação de medidas de } \\
\text { redução do } n^{\circ} \text { de veículos nas } \\
\text { áreas mais poluídas } \\
\text { - Redução de } \mathrm{n}^{\circ} \text { de veículos } \\
\text { circulantes }\end{array}$ \\
\hline SITUAÇÃO & $\begin{array}{l}\text { - Concentração de gases por tipo em } \\
24 \text { horas/bairro } \\
\text { - Ritmo das temperaturas em } 24 \text { horas } \\
\text { - Direção dos ventos/mês }\end{array}$ & $\begin{array}{l}\text { - Avaliação e controle dos níveis } \\
\text { de poluição/qualidade do ar } \\
\text { - Acompanhamento das medidas } \\
\text { para a redução da poluição }\end{array}$ \\
\hline EXPOSIÇÃO & $\begin{array}{l}\text { - Medidas de monitoramento ambiental } \\
\text { para níveis de exposição individual } \\
\text { - Medidas de monitoramento biológico } \\
\text { para indivíduos }\end{array}$ & $\begin{array}{l}\text { - Monitoramento do risco } \\
\text { - Uso de Equipamento de } \\
\text { Proteção Individual } \\
\text { - Programas de orientação }\end{array}$ \\
\hline EFEITO & $\begin{array}{l}\text { - Incidência de internação hospitalar por } \\
\text { tipo de evento e ano }\end{array}$ & - Tratamento de doenças \\
\hline
\end{tabular}

Fonte: II Seminário Nacional de Saúde e Ambiente/FIOCRUZ/2002. 
conferem um outro padrão de poluição. Somam-se a isto as condições climáticas e geográficas, como a direção e a força dos ventos que interferem na dispersão de partículas.

\section{Indicadores de pressão}

Os indicadores de pressão são decorrentes dos indicadores de força motriz. São definidos em parte pelas opções tecnológicas local e regional. Cidades com indústria de química fina, como indústria farmacêutica, e cidades com siderúrgicas tendem a ter padrões de poluição industrial diferentes na emissão de poluentes. O padrão de consumo e o modelo de transporte da região definem as características dos poluentes do ar. O consumo pode se modificar ao longo do tempo e, conseqüentemente, a característica poluidora.

\section{Indicadores de situação}

Relacionam-se neste item as possibilidades de impactos na saúde e no ambiente decorrentes das diversas alterações produzidas no meio ambiente pelas forças motrizes e as pressões. No nosso caso, o ar pode ser afetado alterando o equilíbrio do sistema. $\mathrm{O}$ ar tem uma composição natural que, quando alterada, poderá ou não afetar a saúde e o ambiente. A possibilidade da existência do risco está vinculada ao dano por ele gerado no ambiente e na saúde, e que pode ser a expressão de níveis aceitáveis de um determinado poluente. A concentração ambiental do poluente pode ser um fator que defina a possibilidade da existência de um dano ao ambiente e à saúde.

Os poluentes atmosféricos existem sob a forma de gases e partículas. São denominados contaminantes primários o $\mathrm{CO}, \mathrm{SO} 2$, os NOx e os hidrocarbonetos. Na atmosfera, estes contaminantes se dispersam e sofrem transformações fotoquímicas, produzindo outros contaminantes, denominados secundários, como o ácido sulfúrico (H2SO4), o ácido nítrico (HNO3), diversos nitratos e o ozônio (O3).
Serão apresentados aqui alguns dos principais poluentes do ar que podem provocar agravos na saúde.

\section{- Monóxido de Carbono (CO):}

O CO é um gás tóxico, inodoro, incolor e fruto da combustão de diversos processos industriais, do escapamento de veículos automotores e do fumo do cigarro. A intoxicação aguda pode ser fatal. Sua toxicidade se deve em parte à sua propriedade relacionada à afinidade pela heme da hemoglobina e da mioglobina. O CO, quando absorvido pelo sangue, forma a carboxihemoglobina, que por sua vez produz uma diminuição da oxihemoglobina e uma redução do transporte de oxigênio até os tecidos. O CO possui uma afinidade de até 300 vezes maior com a hemoglobina do que o oxigênio, o que favorece a hipoxemia em pessoas expostas. O CO foi associado a aumento na mortalidade por infarto cardíaco agudo entre as pessoas idosas ${ }^{4}$.

- Óxidos de Nitrogênio (NOx e NO2):

No grupo genérico dos óxidos de nitrogênio (NOx) estão incluídos o dióxido de nitrogênio (NO2), o monóxido de nitrogênio (NO), o ácido nítrico (HNO3) e os nitratos que se formam na combustão de gasolina, carbono e petróleo. Portanto, a principal fonte de produção de NO e NO2 é a emissão de veículos automotores. Existem outras fontes de produção, como a combustão do gás de cozinha e o fumo de cigarro ${ }^{5}$.

\section{- Dióxido de Enxofre (SO2):}

O SO2 é um gás amarelado, com odor característico de enxofre, e muito irritante quando em contato com superfícies úmidas, pois se transforma em ácido sulfúrico. O SO2 é um contaminante produzido por queima de combustível fóssil, escapamento de veículos automotores e subproduto de processos industriais.

A intoxicação pelo $\mathrm{SO} 2$ pode causar irritação da mucosa respiratória desde a nasofaringe e a orofaringe até os alvéolos, levando a inflamação, hemorragia e necrose. A resposta fisiológica primária à inalação do 
SO2 é uma broncoconstricção reflexa e reversível ${ }^{6}$.

- Material Particulado (MP):

$\mathrm{O}$ ar atmosférico contém partículas em suspensão que variam de 0.1 a 10 mm de diâmetro. Partículas maiores que $10 \mathrm{~mm}$ são efetivamente filtradas pelo nariz e pela nasofaringe, onde essas grandes partículas ficam depositadas e podem ser vistas em expectorações e/ou saliva. Partículas menores que 10 mm de diâmetro (MP 10) ficam retidas nas vias aéreas superiores e podem ser depositadas na árvore traqueobrônquica. As partículas menores que 2,5 $\mathrm{mm}$ de diâmetro (MP 2,5) depositam-se no brônquio terminal e nos alvéolos. As concentrações de MP 2,5 representam, em geral, cerca de 45 a $65 \%$ da concentração de MP 10. A fração entre 2,5 e 10 é conhecida como fração grossa do MP 10. A indústria e o transporte são as principais fontes poluidoras de material particulado (MP) ${ }^{7}$.

\section{- Ozônio(O3)}

O ozônio (O3) se forma na atmosfera a partir da reação do oxigênio molecular (O2) com o oxigênio atômico (O-). O NO2, através de uma reação fotoquímica, produz o oxigênio atômico. Portanto, a combinação com o $\mathrm{O} 2$ produz O3. A formação de O3 é típica de áreas urbanas. Normalmente, é um poluente com uma concentração maior em ambientes externos do que em ambientes internos. $\mathrm{OO} 3$ é relativamente pouco solúvel em água e costuma atingir os alvéolos com mais facilidade, produzindo seus efeitos tóxicos nesta região $0^{8}$.

As fontes de emissão são idênticas às de NO2, incluindo veículos automotores e industriais. Os níveis de O3 iniciam sua elevação no período da manhã e têm o seu pico durante à tarde. Os níveis decaem durante o início da noite.

Alguns outros agentes poluidores do ar não foram aqui detalhados. Existem ainda outros exemplos, como o chumbo, presente em alguns produtos, como na gasolina de alguns países e nas tintas industriais, os solventes e os hidrocarbonetos. São metais e substâncias químicas voláteis que podem causar danos durante exposições agudas e crônicas.

\section{Indicadores de exposição}

A exposição humana é a condição necessária para o processo de adoecimento. É importante ressaltar o papel da exposição, compreender os mecanismos envolvidos neste processo e a abordagem metodológica requerida. As questões relacionadas à exposição serão detalhadas em outro tópico deste artigo.

\section{Indicadores de efeito}

Os indicadores deste nível relacionamse diretamente com a saúde da população e dos indivíduos. Estão vinculados ao bem estar e ao padrão de morbi-mortalidade de uma população. $\mathrm{O}$ impacto na saúde, em geral, é o somatório das diversas exposições a diversos agentes, em momentos diferenciados, nem sempre fáceis de serem expostos nos estudos epidemiológicos.

Embora, alguns efeitos crônicos não estejam bem definidos nos indivíduos expostos, os efeitos agudos têm sido mais bem estudados em indivíduos previamente saudáveis, bem como nos portadores de doença respiratória e/ou cardiovascular crônica.

Pode-se separar três tipos principais de efeitos em populações expostas ${ }^{9}$ :

- Efeitos agudos e crônicos em pessoas sadias;

- Efeitos agudos e crônicos em crianças e idosos;

- Exacerbação de doença respiratória preexistente;

- Exacerbação de doença cardíaca préexistente;

- Fenômenos de hipersensibilidade ou de hiperreatividade brônquica não específica.

Nas investigações sobre os efeitos da poluição do ar na saúde, os indicadores de efeitos muitas vezes são obtidos de fontes de dados secundários. Os mais utilizados são os dados de morbidade e de mortalidade por diversas causas. Em geral, este indicador tem um custo mais barato por fazer parte de coletas mais gerais de uma população. Dados primários, como realização de questio- 
nários de sintomas, realização de espirometria e peak-flow em crianças, são úteis para pesquisas que relacionam níveis de poluentes e agravos. Contribuem para construção de um arcabouço legal para os níveis de poluentes e para a compreensão do processo de adoecimento cárdio-respiratório.

Nos estudos de coleta de dados primários para avaliar efeitos de poluentes na saúde, os questionários de sintomas respiratórios têm sido amplamente utilizados. Entretanto, no Brasil não dispomos deste tipo de questionário validado para a nossa população. Outro instrumento importante é o uso da espirometria para avaliar a função pulmonar. Este instrumento começou a fazer parte do arsenal clínico epidemiológico na década de 50 . O exame consiste na medida dos volumes pulmonares e dos fluxos gerados durante ciclos respiratórios basais ou forçados. Permite quantificar a severidade da perda funcional pulmonar e determinar a incapacidade pulmonar.

A medida do pico de fluxo expiratório, conhecido no inglês como peak-flow meter, representa uma outra forma de se estudar o impacto da poluição do ar nos pulmões, principalmente em crianças e idosos, sendo muito utilizada nos estudos de séries temporais. É um aparelho portátil, barato e mede o fluxo expiratório máximo. $\mathrm{O}$ aparelho pode determinar a severidade e a presença de obstrução ao fluxo aéreo, possibilitando uma avaliação rápida do padrão obstrutivo ou de redução dos fluxos expiratórios.

As principais vantagens para o uso do aparelho de pico de fluxo são as medidas diárias de baixo custo, com a possibilidade de detectar alterações diurnas que podem não ser detectadas no momento da espirometria. Além disso, pode ainda identificar exacerbações de doenças de vias aéreas relacionadas aos fatores ambientais.

\section{Avaliação da Exposição em Estudos Epidemiológicos de Poluição do Ar e Saúde}

A Avaliação da Exposição (AE) possui um papel chave quando examinamos os efeitos na saúde decorrentes da poluição do ar. A determinação exata desta exposição é requisito importante para os estudos epidemiológicos e pode fornecer informações tanto sobre a exposição da população a poluentes em ambientes internos (indoor) quanto ao ambiente externo (outdoor). A historia e evolução da AE têm início em 1991, com o relatório do Conselho de Pesquisa Nacional dos Estados Unidos: Avaliação Humana da Exposição aos Poluentes Transportados por via Aérea ${ }^{10}$. O objetivo da $\mathrm{AE}$ aos poluentes do ar é caracterizar as fontes de variação das exposições na população devida a fatores como, por exemplo, lugar e hora do dia, considerando as concentrações dos poluentes nos vários ambientes. Embora as rotas de exposição aos poluentes do ar incluam não somente a inalação, mas também a ingestão e o contato dérmico, neste artigo será focalizada apenas a rota de inalação. O Quadro 2 mostra algumas definições úteis na Avaliação de Exposição.

Como está ilustrado na Figura 1, a AE começa com a identificação das fontes principais dos poluentes selecionados e de suas taxas de emissão no ar. Diversas fontes externas contribuem às emissões de poluentes gasosos e particulados. Similarmente, no ambiente indoor outras fontes e atividades humanas resultam em emissões intermitentes ou contínuas de muitas classes de poluentes, tais como material particulado (MP), monóxido de carbono (CO), compostos orgânicos voláteis (COVs) e agentes biológicos. Estas fontes contribuem para as concentrações de poluentes nos vários microambientes onde a população permanece durante o curso diário de suas atividades.

A exposição à poluição do ar refere-se ao contato com concentrações dos poluentes que um indivíduo encontra no decorrer do tempo (horas, dias, meses etc.) que permanece em ambientes externos e internos. $\mathrm{O}$ poder da fonte pode ser expressa como taxa de emissão (massa por tempo). As unidades são expressas geralmente em $\mu \mathrm{g} / \mathrm{m}^{3} \mathrm{ou} \mathrm{ppm}$. A AE considera que as concentrações podem variar espacial e temporalmente, dependendo da posição e das características 
Quadro 2 - Definições na Avaliação de Exposição (AE)

Chart 2 - Exposure Assessment (EA) definitions

\begin{tabular}{ll}
\hline Termo & Definição \\
\hline Exposição & Exposição a um poluente é um evento que consiste na relação entre o homem e \\
& o ambiente, dada uma concentração específica de poluente ambiental em um \\
& intervalo de tempo determinado. As unidades para expressar a exposição são a \\
& concentração multiplicada pelo tempo. Portanto, o conceito incorpora tanto a \\
& concentração quanto o tempo de duração do contato.
\end{tabular}

Exposição Total do Indivíduo

Abrange todas as exposições a um poluente específico no meio ambiente como um todo (ar, água, alimento e solo) e todas as rotas de entrada (inalação, ingestão e absorção dérmica).

Dose

Dose é a quantidade de poluente absorvida ou depositada no corpo de um organismo exposto por um período de tempo maior - em geral de um único meio. $\mathrm{A}$ dose total é a soma de doses recebidas de um poluente por um indivíduo em um dado intervalo, resultante da interação com todos os meios que apresentem o poluente. Unidades de dose e dose total (massa) são, em geral, convertidas em unidades de massa por volume de fluido fisiológico ou massa por massa de tecido, por exemplo, níveis sangüíneos em $\mu \mathrm{g} / \mathrm{dL}$.

Dose interna Refere-se à quantidade do poluente ambiental absorvido pelo tecido corporal ou à interação com a superfície da membrana de um órgão.

Dose Biologicamente Eficaz É a quantidade do poluente depositado ou absorvido que atinge as células ou o sítio alvo onde surge um efeito adverso ou onde o poluente interage com a superfície da membrana.

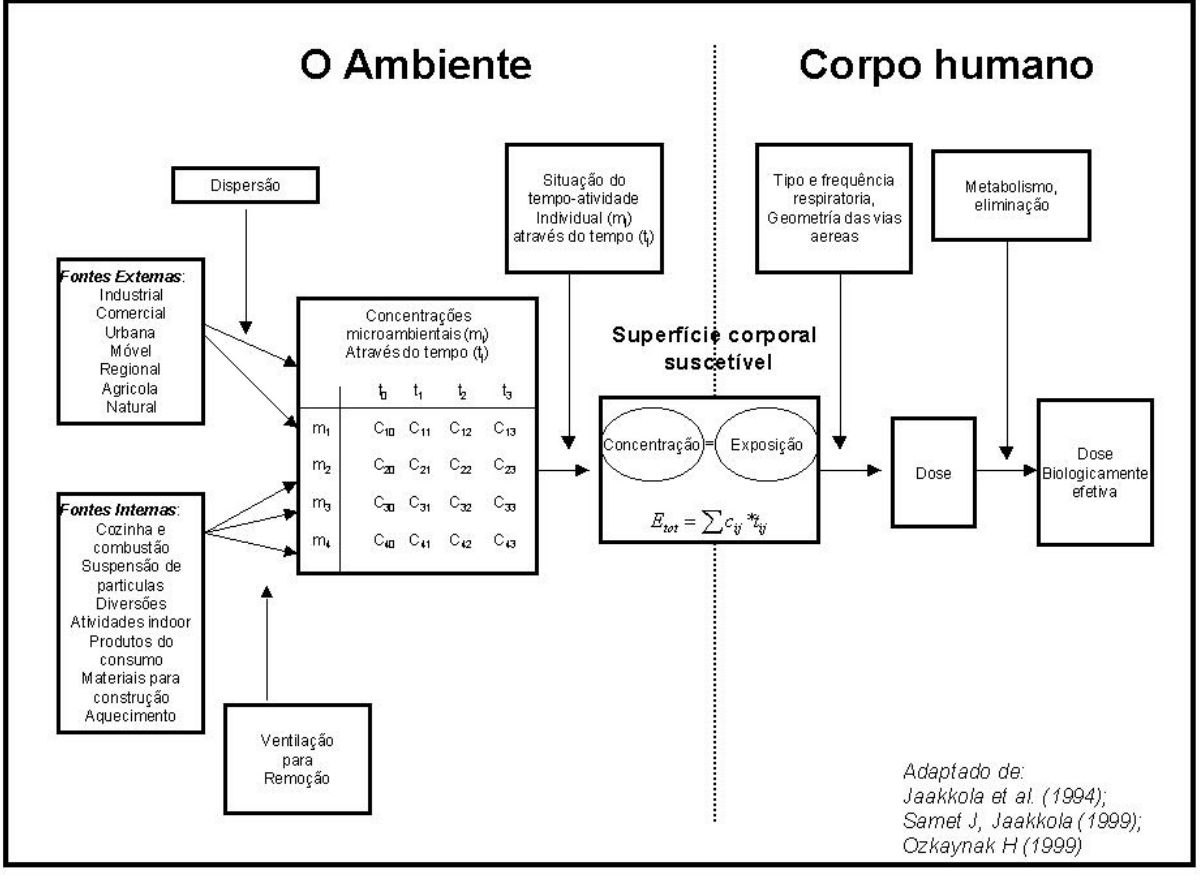

Figura 1 - Identificação das fontes principais dos poluentes selecionados e de suas taxas de emissão no ar.

Figure 1 - Identification of main sources of air pollution assessment. 
das fontes ambientais. A dispersão dos poluentes é influenciada por fatores meteorológicos, tais como a velocidade e o sentido do vento, a temperatura do ar, a umidade relativa e fatores geográficos, como a topografia e a transformação química no ar. Modelos de dispersão têm sido desenvolvidos para se calcular as distribuições da concentração em torno das fontes.

\section{O conceito de microambiente}

O conceito de microambiente foi desenvolvido para auxiliar a avaliação da exposição. Um microambiente é um espaço onde as concentrações dos poluentes de interesse são suficientemente homogêneas para as finalidades de avaliação da exposição. Outros autores consideram um microambiente um "espaço tridimensional onde o nível de poluente em um dado momento é uniforme ou apresenta propriedades estatísticas constantes" ${ }^{11}$. Exemplos de microambientes incluem:

- local de trabalho;

- o quarto durante o sono;

- a cozinha durante o preparo de alimentos (quando os níveis de combustão dos produtos estão elevados) e

- um veículo no trajeto diário da casa para o trabalho.

Microambientes relevantes para avaliação da exposição à poluição do ar são fornecidos na Tabela 1.

Em geral, só os ambientes indoor têm sido considerados como microambientes. No entanto, é muito importante incluir espaços ao ar livre, com concentrações suficientemente homogêneas, como microambientes ${ }^{12}$.

\section{Estratégias gerais para avaliação de exposição}

A exposição pode ser avaliada direta ou indiretamente. No método direto, os indivíduos usam instrumentos (monitores pessoais) que medem a concentração de poluentes na zona de respiração; os monitores podem ser ativos ou passivos. Essa abordagem possui a vantagem de proporcionar um índice de exposição direto e com a possibilidade de elevada validade. Possui a desvantagem de ser, em geral, mais dispendiosa e menos exeqüível do que outras abordagens devido à necessidade de profissionais habilitados no manejo dos equipamentos e da compra de monitores para realização das medições.

No método indireto, o conceito de microambiente é usado para desenvolver estimativas de exposição. Neste método, é necessário informação sobre concentrações de poluentes em diferentes microambientes e os períodos de tempo de permanência em cada microambiente (informações relacionadas ao tempo-atividade). A AE é realizada combinando-se as informações com fórmulas matemáticas. A figura ilustra o conceito e mostra a matriz de concentração-tempo para microambientes diferentes, indoors e outdoors. O símbolo $m_{i}$ refere-se ao número (i) nos diferentes microambientes, $t_{j}$ à concentração de poluente em cada microambiente, e $c_{i j}$ à concentração de poluente em cada microambiente em períodos de tempo específicos. A exposição total integrada de um individuo $\left(E_{\text {to }}\right)$ é estimada multiplicando-se a concentração do poluente em cada microambiente e agregando-se as concentrações ponderadas no tempo:

$$
E_{t o t}=\sum c_{i j}{ }^{*} t_{i j}
$$

\section{Avaliação de tempo-atividade}

A avaliação de tempo-atividade pode ser realizada com métodos retrospectivos ou prospectivos. No método retrospectivo, os padrões de tempo-atividade podem ser avaliados por meio de perguntas relativas a um período de tempo recente (por exemplo, as últimas 24 horas) ou a atividades freqüentes. As perguntas podem se referir ao local em que o tempo é "freqüentemente gasto" ou às atividades de um dia "típico". Listas de microambientes relevantes também podem ser apresentadas, e avaliados a freqüência e a duração do tempo gasto em cada um deles. No método prospectivo, os padrões de tempo-atividade podem ser avaliados com 
Tabela 1 - Microambientes potencialmente importantes para a avaliação da poluição do ar Table 1 - Microenvironments potentially important for air pollution assessment

\begin{tabular}{ll}
\hline Microambientes & Comentários \\
\hline $\begin{array}{l}\text { Ambientes externos } \\
\text { Urbano }\end{array}$ & $\begin{array}{l}\text { Áreas metropolitanas onde os níveis de poluição do ar são altos devido } \\
\text { a uma elevada densidade de fontes móveis e estáticas. }\end{array}$ \\
Cidades de pequeno e médio porte & $\begin{array}{l}\text { Onde os níveis de poluição do ar tendem a ser menores do que em áreas } \\
\text { metropolitanas, embora o transporte da poluição urbana possa afetar a } \\
\text { qualidade do ar local sob certas condições. } \\
\text { Rural } \\
\text { Comunidades agrícolas e cidades pequenas com poucas fontes antropo- } \\
\text { gênicas significantes de poluição do ar, cujos níveis tendem a ser meno- } \\
\text { res; no entanto, o transporte de poluição urbana e suburbana e padrões } \\
\text { regionais de poluição podem afetar a qualidade do ar local em determi- } \\
\text { nadas condições. }\end{array}$ \\
\hline
\end{tabular}

Ambientes internos: Ocupacional

Industrial

Processos de manufatura e produção, tais como os de usinas petroquímicas, moinhos de polpa, usinas hidrelétricas e metalúrgicas.

Não industrial

Indústrias de serviços primários onde os operários não estão envolvidos nos processos de manufatura e de produção, tais como companhias de seguro, escritórios de advocacia e mercados varejistas.

Ambientes internos: Não ocupacional Residencial

Casas com uma só família, apartamentos, casas móveis e condomínios.

Comercial

Restaurantes, lojas de varejo, bancos, supermercados

Público

Correios, tribunais, centros poliesportivos, centros de convenções.

Institucional

Escolas, hospitais, casas de repouso.

Ambientes internos: Transporte

Particular

Automóveis, aviões particulares

Público

Ônibus, metrôs, trens, aviões comerciais.

Fonte/Source: Sexton and Ryan, 1998¹.

técnicas de registros diários em tempo realos indivíduos indicam onde se encontram e o que fazem durante o dia (por exemplo, a cada 15 minutos) ou relatam cada atividade e local, anotando separadamente cada mudança de atividade. Essas técnicas são desenvolvidas para se capturar a informação relativa ao tempo-atividade por meio de mecanismos de registro de dados. Tais mecanismos asseguram que os indivíduos registrem suas atividades da forma como eles as executam no decorrer do dia. Além de coletar informação no âmbito de tempo-atividade, pode ser útil coletá-las também no âmbito da atividade, visto que as relações entre a exposição e a dose podem variar com o grau de atividade para alguns poluentes.
Por exemplo, ao se avaliar os efeitos da exposição ao ozônio na saúde, seria relevante determinar quaisquer atividades externas ocupacionais ou recreativas.

\section{Marcadores biológicos}

Marcadores biológicos são substâncias exógenas ou metabólitos produtos de uma interação entre um agente xenobiótico e uma molécula alvo no corpo, que pode ser quantificada em um compartimento incluindo tecidos, células, fluídos, ou ar expira$\mathrm{do}^{13}$. Os marcadores biológicos podem ser considerados métodos indiretos e AE. Os custos para a determinação destes marcadores variam muito, dependendo da coleta 
de amostras e da complexidade das análises. A carboxihemoglobina é um exemplo de marcador biológico para a exposição ao poluente monóxido de carbono.

É importante lembrar que nas investigações epidemiológicas não temos medições perfeitas. Entretanto, é preciso identificar as possíveis fontes de erro na medição da exposição para conhecer a direção de erro de medida.

\section{Os estudos epidemiológicos}

Estudos epidemiológicos com distintas abordagens têm sido utilizados com o objetivo de se examinar a associação entre a exposição à poluição do ar e seus efeitos na saúde da população. Assim como em outras áreas da investigação epidemiológica, as estratégias básicas empregadas nestes estudos podem ser divididas entre as observacionais e as experimentais ou de intervenção.

Todavia, estudos epidemiológicos experimentais, ou seja, aqueles em que os indivíduos são designados (aleatoriamente ou não) em grupos com diferentes exposições à poluição do ar e então observados os efeitos, são em geral impraticáveis, além de eticamente questionáveis. Experimentos com animais, ou mesmo com voluntários saudáveis, têm se restringido a estudos toxicológicos com pequeno número de participantes, em situações fechadas e controladas, principalmente para determinar níveis máximos toleráveis e alterações anátomo-patológicas ${ }^{14,15}$. Desse modo, a totalidade dos estudos nesta área tem sido do tipo observacional, ou seja, estudos em que o pesquisador apenas observa o curso natural dos eventos, anotando quem é exposto ou não, e quem desenvolveu ou não o desfecho de interesse ${ }^{16}$.

Uma das grandes vantagens dos estudos epidemiológicos para avaliação dos efeitos da poluição na saúde é que eles medem a exposição ambiental à qual, de fato, estamos expostos, além de examinar os efeitos dessa exposição em populações representativas. Conseqüentemente, os resultados podem ser generalizados para a população como um todo. Por outro lado, têm a desvantagem de possibilitar erros de mensuração, principalmente em relação à exposição. Isto porque o caráter ubíquo dessa exposição, que está além do controle individual e tipicamente afeta muitos indivíduos ao mesmo tempo, dificulta muito a obtenção de medidas de exposição individual. A exposição via de regra é medida indiretamente e de maneira agregada, através de uma abordagem ecológica, ou seja, pelos níveis de poluição da área de residência dos indivíduos objetos do estudo.

Medidas de exposição individual à poluição do ar são possíveis, mas o custo dos monitores pessoais e as dificuldades logísticas de tal empreendimento as tornam restritas a estudos com pequeno número de participantes e por períodos de tempo muito restrito ${ }^{17,18}$. Conseqüentemente, estas limitações têm influenciado a escolha do desenho de estudos epidemiológicos. Mais freqüentemente, estudos ecológicos têm sido utilizados, embora estudos longitudinais e, menos freqüentemente, transversais e de caso-controle também venham sendo aplicados nessa área.

\section{Estudos ecológicos}

São estudos em que a unidade de análise não é o indivíduo, mas sim um grupo de indivíduos, ou seja, as informações, tanto sobre a exposição à poluição do ar quanto sobre os indicadores de doença ou evento de interesse, não estão disponíveis individualmente para cada participante do estudo, mas, ao contrário, para um agregado de indivíduos. Os grupos podem ser definidos através de unidades geográficas/espaciais (bairros, cidades, regiões ou países) e a análise baseia-se na comparação dos indicadores de exposição e doença entre as diversas unidades. A evidência de associação é obtida a partir da concordância entre essas variáveis: por exemplo, maiores coeficientes de mortalidade em localidades que apresentam maiores níveis de poluição.

$\mathrm{Na}$ sua grande maioria, esses estudos baseiam-se em informações coletadas roti- 
neiramente e, portanto, podem ser realizados de forma muito rápida e a baixo custo. Por conseguinte, esta abordagem tem sido utilizada em diversos estudos ${ }^{19,20}$, inclusive em nosso meio ${ }^{21}$. Todavia, a possibilidade de erros ou vieses é muito grande devido à dificuldade de se controlar adequadamente para possíveis variáveis de confusão. Além disso, comparações geográficas implicam na utilização de períodos de tempo de maior duração, sendo avaliados principalmente os efeitos crônicos da poluição na saúde.

Os grupos podem também ser definidos através de unidades temporais (anos, meses, dias etc.), e nesse caso podemos chamálo de estudo ecológico temporal. Mais recentemente, com os avanços tecnológicos e computacionais, esse tipo particular de estudo ecológico, também chamado de série temporal, vem sendo amplamente utilizado para avaliação dos efeitos da poluição do ar na saúde. Na série temporal, a unidade de análise é uma unidade de tempo em uma mesma localidade. Como na grande maioria dos casos pretende-se avaliar se exposições agudas aos poluentes do ar estão associadas a efeitos na saúde, séries temporais diárias são utilizadas. Ou seja, coletam-se informações diárias sobre o número de eventos sob investigação (por exemplo, contagem diária de óbitos) e estas são comparadas com os níveis diários dos poluentes.

Uma das grandes vantagens da análise de séries temporais diárias é que fatores como condição sócio-econômica, ocupação ou tabagismo não são capazes de confundir a relação entre a poluição do ar e os efeitos na saúde, uma vez que estes fatores não têm variações diárias. Por outro lado, fatores que apresentam tal variação e estão correlacionados com a poluição são potenciais variáveis de confusão e devem ser ajustados na análise. Entre estes fatores temos as variáveis meteorológicas (temperatura e umidade) e fatores cronológicos ou variáveis relacionadas ao tempo, como dias da semana, estações do ano etc.

Nos últimos anos vem crescendo a quantidade de estudos de séries temporais que vêm investigando a associação da poluição do ar, com os mais variados desfechos. Estes incluem estudos sobre os efeitos na mortalidade geral $^{22}$, na mortalidade por causas específicas como doenças cardiovasculares ${ }^{23}$ ou doenças respiratórias ${ }^{24}$, na morbidade medida por aumentos de sintomas respiratórios em crianças, ou por aumento dos episódios de doença respiratória ${ }^{25}$, ou simplesmente aumento do absenteísmo escolar ${ }^{26}$. Mais recentemente, diversos estudos vêm usando o número de internações hospitalares como um indicador dos efeitos da poluição na saúde da população, utilizando este desenho epidemiológico ${ }^{27,28}$.

\section{Estudos longitudinais}

Em estudos longitudinais ou de coorte, os indivíduos são definidos com base na exposição ou não à poluição do ar e são seguidos por um determinado período de tempo observando-se a ocorrência de alterações no seu status de saúde. Neste caso, portanto, dispõe-se de informações individuais para o estudo das possíveis variáveis de confusão e para o desfecho (morte ou adoecimento). Entretanto, na grande maioria dos casos, a informação sobre exposição continua disponível apenas no âmbito do grupo, baseada nos índices de poluição da área de residência dos indivíduos. Desse modo, estudos com estas características podem também ser considerados estudos híbridos, ou seja, mistos de estudos de coorte e estudos ecológicos.

O mais famoso estudo que aplicou esta metodologia é conhecido como "Estudo das 6 cidades" e foi realizado nos Estados Uni$\operatorname{dos}^{29}$. Neste estudo, uma coorte composta por 8.111 indivíduos de 6 cidades americanas, que apresentavam níveis bastante distintos de poluição do ar, foram acompanhados por um período de 12 a 14 anos. Após ajustes para tabagismo, escolaridade e outros fatores, foram comparados os riscos de mortalidade entre as diferentes categorias de exposição, ou seja, entre as 6 cidades.

Apesar da possibilidade de ajuste para as variáveis de confusão, a validade de estudos desse tipo tem sido questionada dada a im- 
possibilidade de se garantir comparabilidade entre indivíduos residentes em diferentes cidades em relação a inúmeros fatores de risco não controlados, como por exemplo, estilo de vida sedentário ${ }^{30}$. Mesmo assim, a possibilidade do ajuste para diversas outras características no âmbito individual é uma qualidade peculiar deste tipo de abordagem.

Um tipo particular de estudo longitudinal são os chamados estudos de painel, onde um grupo reduzido de indivíduos, idealmente homogêneo, é seguido por um curto espaço de tempo e múltiplas medidas são obtidas de cada sujeito em diversos intervalos no tempo ${ }^{31}$.

Este desenho tem sido empregado para se avaliar a associação de exposição à poluição do ar e a presença de sintomas e sinais respiratórios e/ou mudanças na função pulmonar. Vários destes estudos sugerem um efeito agudo da poluição atmosférica urbana sobre o sistema respiratório, que parece ser mais evidente para pessoas asmáticas do que para indivíduos não asmáticos ${ }^{32,33}$.

Estes estudos apresentam como vantagens o fato de se coletar informações individuais, de não ser necessário recordar eventos para grandes períodos de tempo e, principalmente, o fato de cada indivíduo funcionar como controle de si mesmo. Por outro lado, sofrem dos mesmos problemas associados aos estudos longitudinais.

\section{Outras abordagens}

Outros desenhos epidemiológicos têm sido aplicados, embora menos freqüentemente, nesta área de investigação. Contudo, como já foi dito anteriormente, a definição da exposição dos indivíduos à poluição do ar é quase sempre realizada com o mesmo procedimento, ou seja, indiretamente, através dos níveis medidos na área de residência do indivíduo. Levando em conta esta limitação, estudos transversais ${ }^{34}$ e de caso-controle ${ }^{35}$ têm sido empregados e têm colaborado no estudo dos efeitos adversos à saúde relacionados com a poluição do ar.

\section{Considerações finais}

Os estudos epidemiológicos são úteis para caracterizar a qualidade do ar e os efeitos na saúde. O Brasil precisa acompanhar a tendência mundial no controle dos poluentes do ar e no estímulo à identificação dos agravos dela decorrentes.

Torna-se premente o desenvolvimento de uma estratégia para reduzir o crescimento das emissões de poluentes, como, por exemplo, desestimulando o uso de automóveis nas metrópoles, investindo em transporte público de qualidade, não-poluente, $\mathrm{e}$ encorajando alternativas como o ciclismo.

No setor industrial é necessária uma política de controle e eliminação de resíduos no ar, atuando no sentido do princípio da precaução e reduzindo os efeitos na saúde. No campo, devem ser implementadas a redução de queimadas e uma proposta de agricultura saudável.

É necessária ainda uma atuação conjunta dos três poderes e de todas as esferas do poder público Federal, Estadual e Municipal para garantir a execução de propostas e projetos que reduzam a poluição, possibilitando uma vida mais saudável para a população.

\section{Referências}

1. Belmar R, Hofmeister VA, Michaels D, Moreno AR, Romieu, I. Air Pollutants. In: Finkelman J, Corey G, Calderon R. Environmental Epidemiology: A Project for Latin America and the Caribbean. Metepec, Mexico; 1993. OPAS/WHO. p. 37-94.
2. Corvalán C, Briggs D, Kjellstrom T. Development of Environmental Health Indicators. In: Linkage methods for environmental and health analysis, General Guidelines. A report of the Health and Environment Analysis for Decision-making (HEADLAMP) project. Geneva; (WHO - Office of Global and Integrated Environmental Health); 1996. 
3. Maciel AA, Góes CD, Câncio JA, Oliveira ML, Costa SS. Indicadores de Vigilância Ambiental em Saúde. Informe Epidemiológico do SUS 1999; 8(3): 59-66.

4. Escamilla JÁ. Air Pollution and Fatal Acute Myocardial Infarction in Mexico City: A Case-crossover study. [Ph.D. Thesis]. Baltimore: School of Hygiene and Public Health, Johns Hopkins University; 1999.

5. Linn WS . Controlled exposure of volunteers with chronic obstructive pulmonary disease to nitrogen dioxide. Arch Environ Health 1985; 40: 313-7.

6. Horstman D, Hazucha M, Haak E, Stacy R. Effects of submicronic sulfuric acid aerosol on human pulmonary function. Arch Environ Health 1982; 37: 136-41.

7. World Health Organization (WHO). Quality guidelines for air 1999. World Health Organization. Cluster of sustainable developmnet and health, Geneve 2000.

8. McDonnell W F. Reproducibility of individual responses to ozone exposure. Am Rev Respir Dis 1985; 131: $36-40$.

9. Duchiade MP. Poluição do ar e doenças respiratórias: uma revisão. Cad Saúde Pública 1992; 8(3): 311-30.

10. National Research Council, Committee on Advances in Assessing Human Exposure to Airborne Pollutants Human Exposure Assessment for Airborne Pollutants: Advances and Oportunities. Washington, DC: National Academy Press; 1991.

11. Sexton K, Ryan PB. Assessment of human exposure to air pollution: methods, measurements, and models. In: Watson AY, Bates RR, Kennedy D (eds). Air Pollution, The Automobile and Public Health. Washington, DC: National Academy Press; 1998. p. 207-38.

12. Samet JM, Jaakkola JJ. The Epidemiologic Approach to Investigating Outdoor Air Pollution. In: Holgate ST, Samet JM, Koren HS, Maynard RL (eds). Air Pollution and Health. New York: Academic Press; 1999. p. 431-60.

13. National Research Council. Biologic Markers in Pulmonary Toxicology. Washington, DC: National Academy Press; 1989.

14. Utell MJ, Frampton MW. Toxicologic methods: controlled human exposures. Environ Health Perspect 2000 Aug; 108 (Suppl 4): 605-13.

15. Gilmour MI, Daniels M, McCrillis RC, Winsett D, Selgrade MK. Air pollutant-enhanced respiratory disease in experimental animals. Environ Health Perspect 2001 Aug; 109 (Suppl 4): 619-22.

16. Hennekens CH, Buring JE. Epidemiology in Medicine. Boston: Little, Brown and Company; 1987.

17. Avol EL, Navidi WC, Rappaport EB, Peters JM. Acute effects of ambient ozone on asthmatic, wheezy, and healthy children. Res Rep Health Eff Inst 1998 May; (82): iii, 1-18.
18. Brauer M, Ebelt ST, Fisher TV, Brumm J, Petkau AJ, Vedal S. Exposure of chronic obstructive pulmonary disease patients to particles: respiratory and cardiovascular health effects. J Expo Anal Environ Epidemiol 2001 Nov-Dec; 11(6): 490-500.

19. Bobak M, Leon DA. Air pollution and infant mortality in the Czech Republic, 1986-88. Lancet 1992; 340(8826): 1010-4.

20. Ozkaynak H, Thurston GD. Associations between 1980 US mortality rates and alternative measures of airborne particle concentration. Risk Analysis 1987; 7: 449-61.

21. Penna ML, Duchiade MP. Air pollution and infant mortality from pneumonia in the Rio de Janeiro metropolitan area. Bull Pan Am Health Organ 1991; 25(1): 47-54.

22. Schwartz J. Air pollution and daily mortality in Birmingham, Alabama. Am J Epidemiol 1993; 137: 1136-47.

23. Ballester F, Corella D, Perez Hoyos S, and Hervas A. Air pollution and mortality in Valencia, Spain: a study using the APHEA methodology. J Epidemiol Community Health 1996; 50(5): 527-33.

24. Gouveia N; Fletcher A. Respiratory diseases in children and outdoor air pollution in São Paulo: a time-series analysis. Occup Environ Med 2000; 57: 477-83.

25. Pope CA, Dockery DW. Acute health effects of PM10 pollution on symptomatic and asymptomatic children. Am Rev Respir Dis 1992; 145(5): 1123-8.

26. Romieu I, Lugo MC, Velasco SR, Sanchez S, Meneses F, Hernandez M. Air pollution and school absenteeism among children in Mexico City. Am J Epidemiol 1992; 136(12): 1524-31.

27. Schwartz J. Air pollution and hospital admissions for respiratory disease. Epidemiology 1996; 7: 20-8.

28. Gouveia N, Fletcher A. Time Series Analysis of Air Pollution and Mortality: Effects by cause, age and socioeconomic status. J Epidemiol Community Health 2000; 54: 750-5.

29. Dockery DW, Pope AC, Xu X et al. An association between air pollution and mortality in six U.S. cities. $N$ Engl J Med 1993; 329: 1753-9.

30. Moolgavkar SH, Luebeck EG. A critical review of the evidence on particulate air pollution and mortality. Epidemiology 1996; 7(4): 420-8.

31. Kelsey JL, Thompson WD, Evans A. Methods in observational epidemiology. New York: Oxford University Press; 1986.

32. Yu O, Sheppard L, Lumley T, Koenig JQ, Shapiro GG. Effects of ambient air pollution on symptoms of asthma in Seattle-area children enrolled in the CAMP study. Environ Health Perspect. 2000 Dec; 108(12): 1209-14. 
33. Roemer W, Hoek G, Brunekreef B, Haluszka J, Kalandidi A, Pekkanen J. Daily variations in air pollution and respiratory health in a multicentre study: the PEACE project. Pollution Effects on Asthmatic Children in Europe. Eur Respir J 1998 Dec; 12(6): 1354-61.

34. Sobral HR. Air pollution and respiratory diseases in children in São Paulo, Brazil. Soc Sci Med 1989; 29(8): 959-64.
35. Lin S, Munsie JP, Hwang SA, Fitzgerald E, Cayo MR. Childhood asthma hospitalization and residential exposure to state route traffic. Environ Res. 2002 Feb; 88(2): 73-81.

Recebido em: 24/07/2002

Aprovação em: 29/10/2002 